\title{
A note on differential-difference analogue of Brück conjecture
}

\author{
Minfeng Chen ${ }^{\mathrm{a}, *}$, Ning Cui ${ }^{\mathrm{b}}$ \\ a School of Mathematics and Statistics, Guangdong University of Foreign Studies, Guangzhou 510006, \\ China \\ b College of Mathematics and Information Science, Zhengzhou University of Light Industry, Zhengzhou \\ 450002, China
}

*Corresponding author, e-mail: chenminfeng198710@126.com

Received 1 Mar 2019

Accepted 7 Aug 2019

ABSTRACT: In this paper, we prove that for a transcendental entire function $f(z)$ of finite order, $\eta \in \mathbb{C} \backslash\{0\}$ is a constant such that $\Delta_{\eta} f(z)=f(z+\eta)-f(z) \not \equiv 0, b(z)$ is an entire function such that $\sigma(b)<\sigma(f)$ and $\lambda(f-b)<\sigma(f)$, if $\Delta_{\eta} f(z)$ and $f^{\prime}(z)$ share $a(z) \mathrm{CM}$, where $a(z)$ is an entire function satisfying $\sigma(a)<\sigma(f)$, then

$$
\frac{\Delta_{\eta} f(z)-a(z)}{f^{\prime}(z)-a(z)}=A, \quad f(z)=b(z)+H(z) \mathrm{e}^{c z},
$$

where $a(z)$ and $b(z)$ are entire functions with $\max \{\sigma(a), \sigma(b)\}<1, H(z)(\not \equiv 0)$ is an entire function with $\lambda(H)=$ $\sigma(H)<1$ and $A, c, \eta \in \mathbb{C} \backslash\{0\}$ are constants satisfying $\mathrm{e}^{c \eta}=1+A c$. Our results are improvements and complements of those in [Bull Korean Math Soc 51 (2014) 1453-1467] and [Commun Korean Math Soc 32 (2017) 361-373].

KEYWORDS: entire function, sharing value, differential-difference equation

MSC2010: 39B32 34M05 30D35

\section{INTRODUCTION}

In this paper, we assume that the reader is familiar with the standard symbols and the fundamental results of Nevanlinna theory ${ }^{1-3}$. In addition, we use the notations $\lambda(f)$ and $\sigma(f)$ to denote the exponent of convergence of the zero sequence and the order of growth of meromorphic function $f(z)$, respectively. We also denote by $S(r, f)$ any quantify satisfying $S(r, f)=o(T(r, f))$, as $r \rightarrow \infty$, outside of a possible exceptional set of finite logarithmic measure. For convenience, we need the following definition.

Let $f(z)$ and $g(z)$ be two non-constant meromorphic functions, and let $a$ be a constant in the complex plane. We say that $f(z)$ and $g(z)$ share $a$ CM (IM) provided that $f(z)-a$ and $g(z)-a$ have the same zeros counting multiplicities (ignoring multiplicities), and $f(z)$ and $g(z)$ share $\infty$ CM (IM) provided that $f(z)$ and $g(z)$ have the same poles counting multiplicities (ignoring multiplicities). Using the same method, we can also define $f(z)$ and $g(z)$ share function $a(z)$ CM (IM), where $a(z) \in$ $S(r, f) \cap S(r, g)$.

Definition 1 (Ref. 4) Let $f(z)$ be a meromorphic function in the complex plane. We denote by $\sigma_{2}(f)$ the order of $\log T(r, f)$, i.e.,

$$
\sigma_{2}(f)=\limsup _{r \rightarrow \infty} \frac{\log \log T(r, f)}{\log r} .
$$

$\sigma_{2}(f)$ is called the hyper-order of $f(z)$.

Brück ${ }^{5}$ raised the following conjecture.

Conjecture (Ref. 5) Let $f(z)$ be a non-constant entire function with hyper-order $\sigma_{2}(f)<\infty$, and $\sigma_{2}(f) \notin \mathbb{Z}^{+}$. If $f(z)$ and $f^{\prime}(z)$ share a finite value $a$ $\mathrm{CM}$, then

$$
\frac{f^{\prime}(z)-a}{f(z)-a}=c,
$$

where $c$ is a non-zero constant.

The conjecture has been established in the special case ${ }^{5}$ when $a=0$ or when $f(z)$ is an entire function of finite order ${ }^{6}$.

Recently, many results on difference analogues of Brück conjecture were considered in Refs. 7-12. To start with, recall the following results.

Theorem 1 (Ref. 9) Let $f(z)$ be a meromorphic function of $\sigma(f)<2$, and $\eta$ be a non-zero constant. 
If $f(z)$ and $f(z+\eta)$ share a finite value $a$ and $\infty C M$, then

$$
\frac{f(z+\eta)-a}{f(z)-a}=\tau,
$$

for some constant $\tau$.

Heittokangas et $\mathrm{al}^{9}$ gave the example $f(z)=$ $\mathrm{e}^{z^{2}}+1$ which shows that $\sigma(f)<2$ cannot be relaxed to $\sigma(f) \leqslant 2$.

It is well known that $\Delta_{\eta} f(z)=f(z+\eta)-f(z)$ (where $\eta \in \mathbb{C} \backslash\{0\}$ is a constant such that $f(z+\eta)$ $f(z) \not \equiv 0)$ is regarded as the difference counterpart of $f^{\prime}(z)$. For a transcendental entire function $f(z)$ with finite order which has a finite Borel exceptional value, Chen and $\mathrm{Yi}^{7}$ and $\mathrm{Chen}^{8}$ proceed to consider the problem that $\Delta_{\eta} f(z)$ and $f(z)$ share one finite value $\mathrm{CM}$ and have obtained the following results.

Theorem 2 (Ref. 7) Let $f(z)$ be a finite-order transcendental entire function which has a finite Borel exceptional value $a$, and let $\eta$ be a constant such that $f(z+\eta) \not \equiv f(z)$. If $\Delta_{\eta} f(z)$ and $f(z)$ share $a C M$, then

$$
a=0 \text { and } \frac{f(z+\eta)-f(z)}{f(z)}=c
$$

for some constant $c$.

Theorem 3 (Ref. 8) Let $f(z)$ be a transcendental entire function of finite order that is of a finite Borel exceptional value $\alpha$, and $\eta \in \mathbb{C}$ be a constant such that $f(z+\eta) \not \equiv f(z)$. If $\Delta_{\eta} f(z)=f(z+\eta)-f(z)$ and $f(z)$ share $a(\neq \alpha) C M$, then

$$
\frac{\Delta_{\eta} f(z)-a}{f(z)-a}=\frac{a}{a-\alpha} .
$$

After that Liu and Dong ${ }^{13}$ considered the differential-difference analogue of Brück conjecture and have obtained the following result.

Theorem 4 (Ref. 13) Suppose that $f(z)$ is an entire solution of equation

$$
f^{\prime}(z)-a(z)=\mathrm{e}^{P(z)}(f(z+c)-a(z)),
$$

where $c \in \mathbb{C} \backslash\{0\}$ is a constant, $P(z)$ is a polynomial and $a(z)$ is an entire function with $\sigma(a)<\sigma(f)$. If $\lambda(f-a)<\sigma(f)$, then $\sigma(f)=1+\operatorname{deg} P(z)$.

Chen and $\mathrm{Gao}^{14}$ have recently proved the following result.

Theorem 5 (Ref. 14) Let $f(z)$ be a transcendental entire function of finite order, $\eta \in \mathbb{C} \backslash\{0\}$ be a constant such that $\Delta_{\eta} f(z)=f(z+\eta)-f(z) \not \equiv 0, a(z)$ be an entire function such that $\sigma(a)<1$ and $\lambda(f-a)<$ $\sigma(f)$. If $\Delta_{\eta} f(z)$ and $f^{\prime}(z)$ share $a(z) C M$, then one of the following two cases holds: (i) If $a(z) \not \equiv 0$, then

$$
\frac{\Delta_{\eta} f(z)-a(z)}{f^{\prime}(z)-a(z)}=1, \quad f(z)=a(z)+H(z) \mathrm{e}^{c z},
$$

where $H(z) \not \equiv 0$ is an entire function with $\lambda(H)=\sigma(H)<1$ and $c \in \mathbb{C} \backslash\{0\}$ is a constant satisfying $\mathrm{e}^{c \eta}=1+c$;

(ii) If $a(z) \equiv 0$, then

$$
\frac{\Delta_{\eta} f(z)}{f^{\prime}(z)}=A, \quad f(z)=H(z) \mathrm{e}^{c z},
$$

where $H(z) \not \equiv 0$ is an entire function with $\lambda(H)=\sigma(H)<1, A, c \in \mathbb{C} \backslash\{0\}$ are constants satisfying $\mathrm{e}^{c \eta}=1+A c$.

\section{RESULTS}

Here, we will proceed to consider the differentialdifference analogue of Brück conjecture and obtain the accurate expression of the transcendental entire function $f(z)$. The aim of this paper is to improve the results obtained in Theorem 4 and Theorem 5. In fact, we will prove the following result.

Theorem 6 Let $f(z)$ be a transcendental entire function of finite order, $\eta \in \mathbb{C} \backslash\{0\}$ be a constant such that $\Delta_{\eta} f(z)=f(z+\eta)-f(z) \not \equiv 0, b(z)$ be an entire function such that $\sigma(b)<\sigma(f)$ and $\lambda(f-b)<\sigma(f)$. If $\Delta_{\eta} f(z)$ and $f^{\prime}(z)$ share $a(z) C M$, where $a(z)$ is an entire function satisfying $\sigma(a)<\sigma(f)$, then

$$
\frac{\Delta_{\eta} f(z)-a(z)}{f^{\prime}(z)-a(z)}=A, \quad f(z)=b(z)+H(z) \mathrm{e}^{c z},
$$

where $a(z), b(z)$ are entire functions with $\max \{\sigma(a), \sigma(b)\}<1, \quad H(z) \not \equiv 0$ is an entire function with $\lambda(H)=\sigma(H)<1$ and $A, c, \eta \in \mathbb{C} \backslash\{0\}$ are constants satisfying $\mathrm{e}^{c \eta}=1+A c$.

Remark 1 From the assumptions of Theorem 6, we conclude that $\sigma(f) \geqslant 1$. Hence if $\sigma(a)<1$ and $\sigma(b)<1$, we obtain the following corollary.

Corollary 1 Let $f(z)$ be a transcendental entire function of finite order, $\eta \in \mathbb{C} \backslash\{0\}$ be a constant such that $\Delta_{\eta} f(z)=f(z+\eta)-f(z) \not \equiv 0, b(z)$ be an entire function such that $\sigma(b)<1$ and $\lambda(f-b)<\sigma(f)$. If $\Delta_{\eta} f(z)$ and $f^{\prime}(z)$ share $a(z) C M$, where $a(z)$ is an entire function satisfying $\sigma(a)<1$, then

$$
\frac{\Delta_{\eta} f(z)-a(z)}{f^{\prime}(z)-a(z)}=A, \quad f(z)=b(z)+H(z) \mathrm{e}^{c z},
$$

where $H(z) \not \equiv 0$ is an entire function with $\lambda(H)=$ $\sigma(H)<1$ and $A, c, \eta \in \mathbb{C} \backslash\{0\}$ are constants satisfying $\mathrm{e}^{c \eta}=1+A c$. 
Remark 2 From the assumptions of Theorem 6, we conclude that $\sigma(f) \geqslant 1$. Hence if $a(z) \equiv b(z) \not \equiv 0$ and $\sigma(a)<1$, we obtain the following corollary, which is the conclusion (i) of Theorem 5.

Corollary 2 Let $f(z)$ be a transcendental entire function of finite order, $\eta \in \mathbb{C} \backslash\{0\}$ be a constant such that $\Delta_{\eta} f(z)=f(z+\eta)-f(z) \not \equiv 0, a(z)$ be an entire function such that $\sigma(a)<1$ and $\lambda(f-a)<\sigma(f)$. If $\Delta_{\eta} f(z)$ and $f^{\prime}(z)$ share $a(z) \not \equiv 0 C M$, then

$$
\frac{\Delta_{\eta} f(z)-a(z)}{f^{\prime}(z)-a(z)}=A, \quad f(z)=a(z)+H(z) \mathrm{e}^{c z},
$$

where $H(z) \not \equiv 0$ is an entire function with $\lambda(H)=$ $\sigma(H)<1$ and $A, c, \eta \in \mathbb{C} \backslash\{0\}$ are constants satisfying $\mathrm{e}^{c \eta}=1+A c$.

Remark 3 In Theorem 6, if $b(z) \equiv b$ and $a(z) \equiv a$, we obtain the following corollary.

Corollary 3 Let $f(z)$ be a transcendental entire function of finite order which has a finite Borel exceptional $b, \eta \in \mathbb{C} \backslash\{0\}$ be a constant such that $\Delta_{\eta} f(z)=$ $f(z+\eta)-f(z) \not \equiv 0$. If $\Delta_{\eta} f(z)$ and $f^{\prime}(z)$ share $a \neq 0$ $C M$, then

$$
\frac{\Delta_{\eta} f(z)-a}{f^{\prime}(z)-a}=A, \quad f(z)=b+H(z) \mathrm{e}^{c z},
$$

where $H(z) \not \equiv 0$ is an entire function with $\lambda(H)=$ $\sigma(H)<1, A, c, \eta \in \mathbb{C} \backslash\{0\}$ are constants satisfying $\mathrm{e}^{c \eta}=1+A c$.

Examples 1, 2, and 3 below show that Corollaries 1,2 , and 3 are sharp, respectively.

Example 1 (Ref. 14) Suppose that $f(z)=z^{2}+\mathrm{e}^{c z}$, where $c \in \mathbb{C} \backslash\{0\}$ is a constant. Then $\lambda\left(f-z^{2}\right)<$ $\sigma(f)$. Let $\eta=1$ and let $c$ satisfy $\mathrm{e}^{c}=1+\frac{1}{2} c$, we see that $\Delta_{\eta} f(z)=2 z+1+\frac{1}{2} c \mathrm{e}^{c z}$ and $f^{\prime}(z)=2 z+c \mathrm{e}^{c z}$. Then $\left(\Delta_{\eta} f(z)-2(z+1)\right) /\left(f^{\prime}(z)-2(z+1)\right)=\frac{1}{2}$, that is, $\Delta_{\eta} f(z)$ and $f^{\prime}(z)$ share $2(z+1)\left(\not \equiv z^{2}\right)$ CM.

Example 2 (Ref. 14) Suppose that $f(z)=z+\mathrm{e}^{c z}$, where $c \in \mathbb{C} \backslash\{0\}$ is a constant. Then $\lambda(f-z)<$ $\sigma(f)$. Let $\eta=1$ and let $c$ satisfy $\mathrm{e}^{c}=1+c$, we see that $\Delta_{\eta} f(z)=1+c \mathrm{e}^{c z}=f^{\prime}(z)$. Then $\left(\Delta_{\eta} f(z)-\right.$ $z) /\left(f^{\prime}(z)-z\right)=1$, that is, $\Delta_{\eta} f(z)$ and $f^{\prime}(z)$ share $z$ CM.

Example 3 Suppose that $f(z)=1+\mathrm{e}^{c z}$, where $c \in$ $\mathbb{C} \backslash\{0\}$ is a constant. Then $\lambda(f-1)<\sigma(f)$. Let $\eta=\log 2$ and let $c$ satisfy $2^{c}=1+c$, we see that $\Delta_{\eta} f(z)=c \mathrm{e}^{c z}=f^{\prime}(z)$. Then $\left(\Delta_{\eta} f(z)-1\right) /\left(f^{\prime}(z)-\right.$ $1)=1$; that is, $\Delta_{\eta} f(z)$ and $f^{\prime}(z)$ share $1 \mathrm{CM}$.

\section{SOME LEMMAS}

Lemma 1 (Ref. 15, Corollary 2) Let $f(z)$ be a transcendental meromorphic function of finite order $\sigma$, let $k, j(k>j \geqslant 0)$ be integers. Then for any given $\varepsilon>0$, there exists a set $E \subset(1, \infty)$ of finite logarithmic measure, such that for all $z$ satisfying $|z|=r \notin[0,1] \cup E$, we have

$$
\left|\frac{f^{(k)}(z)}{f^{(j)}(z)}\right| \leqslant|z|^{(k-j)(\sigma-1+\varepsilon)} .
$$

Lemma 2 (Ref. 16, Theorem 8.2) Let $f(z)$ be a meromorphic function of finite order $\sigma$, let $\eta$ be a non-zero complex number, and let $\varepsilon>0$ be a given real constant. Then there exists a subset $E \subset(1, \infty)$ of finite logarithmic measure such that for all $|z|=$ $r \notin[0,1] \cup E$, we have

$$
\exp \left\{-r^{\sigma-1+\varepsilon}\right\} \leqslant\left|\frac{f(z+\eta)}{f(z)}\right| \leqslant \exp \left\{r^{\sigma-1+\varepsilon}\right\} .
$$

Following Hayman (Ref. 17), we define an $\varepsilon$-set to be a countable union of open discs not containing the origin and subtending angles at the origin whose sum is finite. If $E$ is an $\varepsilon$-set, then the set of $r \geqslant 1$ for which the circle $S(0, r)$ meets $E$ has finite logarithmic measure, and for almost all real $\theta$ the intersection of $E$ with the ray $\arg z=\theta$ is bounded.

Lemma 3 (Ref. 18, Lemma 3.3) Let $f(z)$ be a transcendental meromorphic function of order $\sigma(f)<1$, and let $h>0$. There exists an $\varepsilon$-set $E$ such that

$$
\begin{aligned}
\frac{f^{\prime}(z+c)}{f(z+c)} & \rightarrow 0, \quad \frac{f(z+c)}{f(z)} \rightarrow 1 \\
\text { as } z & \rightarrow \infty \text { in } \mathbb{C} \backslash E,
\end{aligned}
$$

uniformly in $c$ for $|c| \leqslant h$. Further, $E$ may be chosen so that for large $z \notin E$, the function $f(z)$ has no zeros or poles on $|\zeta-z| \leqslant h$.

Lemma 4 (Ref. 4) Suppose that $f_{j}(z) \quad(j=$ $1,2, \ldots, n+1)$ and $g_{j}(z)(j=1,2, \ldots, n)(n \geqslant 1)$ are entire functions satisfying (i) $\sum_{j=1}^{n} f_{j}(z) \mathrm{e}^{g_{j}(z)} \equiv$ $f_{n+1}(z)$; (ii) The order of $f_{j}(z)$ is less than the order of $\mathrm{e}^{g_{k}(z)}$ for $1 \leqslant j \leqslant n+1,1 \leqslant k \leqslant n$; and furthermore, the order of $f_{j}(z)$ is less than the order of $\mathrm{e}^{g_{h}(z)-g_{k}(z)}$ for $n \geqslant 2$ and $1 \leqslant j \leqslant n+1,1 \leqslant h<k \leqslant n$. Then $f_{j}(z) \equiv 0,(j=1,2, \ldots, n+1)$.

Lemma 5 Let $f(z)$ be a transcendental entire function of finite order, $\eta \in \mathbb{C} \backslash\{0\}$ be a constant such that $\Delta_{\eta} f(z)=f(z+\eta)-f(z) \not \equiv 0, b(z)$ be an entire 
function such that $\sigma(b)<\sigma(f)$ and $\lambda(f-b)<\sigma(f)$. If

$$
\frac{\Delta_{\eta} f(z)-a(z)}{f^{\prime}(z)-a(z)}=A,
$$

where $A \in \mathbb{C} \backslash\{0\}$ is a constant and $a(z)$ is an entire function such that $\sigma(a)<\sigma(f)$, then

$$
f(z)=b(z)+H(z) \mathrm{e}^{c z},
$$

where $b(z)$ is an entire function with $\sigma(b)<1$, $H(z) \not \equiv 0$ is an entire function with $\lambda(H)=\sigma(H)<1$ and $A, c, \eta \in \mathbb{C} \backslash\{0\}$ are constants satisfying $\mathrm{e}^{c \eta}=$ $1+A c$.

Proof: By Hadamard's factorization theorem (Ref. 4, Theorem 2.5), we obtain

$$
f(z)=b(z)+h(z) \mathrm{e}^{Q(z)},
$$

where $h(z) \not \equiv 0$ is an entire function, $Q(z)$ is a polynomial with $\operatorname{deg} Q(z)=q \geqslant 1$, and $h(z), Q(z)$ satisfy

$$
\sigma(h)=\lambda(h)=\lambda(f-b)<\sigma(f)=\operatorname{deg} Q(z) .
$$

Note that

$$
\frac{\Delta_{\eta} f(z)-a(z)}{f^{\prime}(z)-a(z)}=A .
$$

Substituting (1) into (3) yields

$$
\begin{aligned}
h(z+\eta) \mathrm{e}^{Q(z+\eta)-Q(z)}-h(z)-A\left(h^{\prime}(z)\right. \\
\left.+h(z) Q^{\prime}(z)\right)=(A d(z)-c(z)) \mathrm{e}^{-Q(z)},
\end{aligned}
$$

where $c(z)=b(z+\eta)-b(z)-a(z)$ and $d(z)=$ $b^{\prime}(z)-a(z)$. Since $\sigma(a)<q$ and $\sigma(b)<q$, we see that $\max \{\sigma(c), \sigma(d)\}<q$. If $\operatorname{Ad}(z)-c(z) \not \equiv$ 0 , since $\sigma(h)<q, \operatorname{deg}(Q(z+\eta)-Q(z))=q-1$ and $\max \{\sigma(c), \sigma(d)\}<q$, we see that the order of growth of the left side of (4) is less than $q$, and the order of growth of the right side of (4) is $q$, a contradiction. Then $\operatorname{Ad}(z)-c(z) \equiv 0$, (4) can be rewritten as

$$
\mathrm{e}^{Q(z+\eta)-Q(z)}=\left[1+A\left(\frac{h^{\prime}(z)}{h(z)}+Q^{\prime}(z)\right)\right] \frac{h(z)}{h(z+\eta)} .
$$

We claim that $q=1$. In fact, if it is not true, then $q \geqslant$ 2. If $\sigma(h)<1$, since $\operatorname{deg}(Q(z+\eta)-Q(z))=q-1 \geqslant 1$, we see that the order of growth of the left side of (5) is $q-1 \geqslant 1$, and the order of growth of the right side of (5) is less than 1 , a contradiction. Then we have $\sigma(h) \geqslant 1$.
By Lemma 1 , for any given $\varepsilon_{1}>0$, there exists a set $E_{1} \subset(1, \infty)$ of finite logarithmic measure, such that for all $z$ satisfying $|z|=r \notin[0,1] \cup E_{1}$, we have

$$
\left|\frac{h^{\prime}(z)}{h(z)}\right| \leqslant|z|^{\sigma(h)-1+\varepsilon_{1}} .
$$

By Lemma 2, for any given $\varepsilon_{2}>0$, there exists a set $E_{2} \subset(1, \infty)$ of finite logarithmic measure, such that for all $z$ satisfying $|z|=r \notin[0,1] \cup E_{2}$, we have

$$
\exp \left\{-r^{\sigma(h)-1+\varepsilon_{2}}\right\} \leqslant\left|\frac{h(z+\eta)}{h(z)}\right| \leqslant \exp \left\{r^{\sigma(h)-1+\varepsilon_{2}}\right\} .
$$

Set $\varepsilon_{3}=\max \left\{\varepsilon_{1}, \varepsilon_{2}\right\}, 0<\varepsilon_{3}<\frac{1}{3}(q-\sigma(h))$, there exists $r_{0}>0$ such that for all $z$ satisfying $|z|=r>r_{0}$, we have

$$
r^{q-1-\varepsilon_{3}} \leqslant\left|Q^{\prime}(z)\right| \leqslant r^{q-1+\varepsilon_{3}} .
$$

From (5), we see that $\left(1+A\left(h^{\prime}(z) / h(z)+\right.\right.$ $\left.\left.Q^{\prime}(z)\right)\right) h(z) / h(z+\eta)$ is an entire function. Then for all $z$ satisfying $|z|=r>r_{0}$ and $|z|=r \notin[0,1] \cup E_{1} \cup$ $E_{2}$, for the above given $\varepsilon_{3}$, from (6)-(8), we have

$$
\begin{aligned}
\mid(1+ & \left.A\left(\frac{h^{\prime}(z)}{h(z)}+Q^{\prime}(z)\right)\right) \frac{h(z)}{h(z+\eta)} \mid \\
& \leqslant\left(1+|A|\left(\left|\frac{h^{\prime}(z)}{h(z)}\right|+\left|Q^{\prime}(z)\right|\right)\right)\left|\frac{h(z)}{h(z+\eta)}\right| \\
& \leqslant\left(1+|A|\left(r^{\sigma(h)-1+\varepsilon_{3}}+r^{q-1+\varepsilon_{3}}\right)\right) \exp \left\{r^{\sigma(h)-1+\varepsilon_{3}}\right\} \\
& \leqslant|A| r^{\sigma(h)+q-2+2 \varepsilon_{3}} \exp \left\{r^{\sigma(h)-1+\varepsilon_{3}}\right\}<\exp \left\{r^{q-1}\right\},
\end{aligned}
$$

that is,

$$
\begin{aligned}
T(r,(1 & \left.\left.+A\left(\frac{h^{\prime}(z)}{h(z)}+Q^{\prime}(z)\right)\right) \frac{h(z)}{h(z+\eta)}\right) \\
& =m\left(r,\left(1+A\left(\frac{h^{\prime}(z)}{h(z)}+Q^{\prime}(z)\right)\right) \frac{h(z)}{h(z+\eta)}\right) \\
& <r^{q-1}
\end{aligned}
$$

The above inequality yields

$$
\sigma\left(\left(1+A\left(\frac{h^{\prime}(z)}{h(z)}+Q^{\prime}(z)\right)\right) \frac{h(z)}{h(z+\eta)}\right)<q-1 .
$$

It follows from $\operatorname{deg}(Q(z+\eta)-Q(z))=q-1$ that (5) is a contradiction. Then we must have $q=1$,

$$
f(z)=b(z)+H(z) \mathrm{e}^{c z},
$$

where $c \in \mathbb{C} \backslash\{0\}$ is a constant and $H(z) \not \equiv 0$ is an entire function with $\lambda(H)=\sigma(H)<1$. It follows from (5) that

$$
\frac{h(z+\eta)}{h(z)} \mathrm{e}^{c \eta}=1+A\left(\frac{h^{\prime}(z)}{h(z)}+c\right) .
$$


If $h(z) \not \equiv 0$ is a polynomial, then

$$
\frac{h^{\prime}(z)}{h(z)} \rightarrow 0, \quad \frac{h(z+\eta)}{h(z)} \rightarrow 1, \quad z \rightarrow \infty .
$$

It follows from (9) and (10) that $\mathrm{e}^{c \eta}=1+A c$. If $h(z) \not \equiv 0$ is a transcendental entire function with $\sigma(h)<1$, from Lemma 3, we also have $\mathrm{e}^{c \eta}=1+$ Ac.

\section{PROOF OF Theorem 6}

Proof: From the assumptions of Theorem 6, we see that (1) and (2) are still valid. Since $\Delta_{\eta} f(z)$ and $f^{\prime}(z)$ share $a(z) \mathrm{CM}$, we have

$$
\frac{\Delta_{\eta} f(z)-a(z)}{f^{\prime}(z)-a(z)}=\mathrm{e}^{P(z)},
$$

where $P(z)$ is a polynomial. It follows from (2) and (11) that

$$
\operatorname{deg} P(z) \leqslant \operatorname{deg} Q(z) .
$$

Substituting (1) into (11) yields

$$
\begin{aligned}
h(z+\eta) \mathrm{e}^{Q(z+\eta)-Q(z)}-h(z)+c(z) \mathrm{e}^{-Q(z)} & \\
= & \left(h^{\prime}(z)+h(z) Q^{\prime}(z)+d(z) \mathrm{e}^{-Q(z)}\right) \mathrm{e}^{P(z)},
\end{aligned}
$$

where $c(z)=b(z+\eta)-b(z)-a(z)$ and $d(z)=b^{\prime}(z)-$ $a(z)$. Since $\sigma(a)<\sigma(f)$ and $\sigma(b)<\sigma(f)$, we see that $\max \{\sigma(c), \sigma(d)\}<\sigma(f)$. In what follows, we consider two cases: $1 \leqslant \operatorname{deg} P(z)<\operatorname{deg} Q(z)$ and $\operatorname{deg} P(z)=\operatorname{deg} Q(z)$. Set

$$
\begin{aligned}
& P(z)=a_{p} z^{p}+a_{p-1} z^{p-1}+\cdots+a_{0}, \\
& Q(z)=b_{q} z^{q}+b_{q-1} z^{q-1}+\cdots+b_{0},
\end{aligned}
$$

where $a_{p}(\neq 0), \ldots, a_{0}, b_{q}(\neq 0), \ldots, b_{0}$ are constants, $p, q$ are positive integers.

Case 1. Suppose that $1 \leqslant p<q$. Then (13) can be rewritten as

$$
\begin{array}{r}
h(z+\eta) \mathrm{e}^{Q(z+\eta)-Q(z)}-h(z)-\left(h^{\prime}(z)+h(z) Q^{\prime}(z)\right) \mathrm{e}^{P(z)} \\
=\left(d(z) \mathrm{e}^{P(z)}-c(z)\right) \mathrm{e}^{-Q(z)} .
\end{array}
$$

If $d(z) \mathrm{e}^{P(z)}-c(z) \not \equiv 0$, since $\sigma(h)<q, \operatorname{deg}(Q(z+\eta)-$ $Q(z))=q-1$ and $\sigma\left(\mathrm{e}^{P(z)}\right)=\operatorname{deg} P(z)=p<q$, we see that the order of growth of the left side of (5) is less than $q$, and the order of growth of the right side of (5) is $q$, a contradiction. If $d(z) \mathrm{e}^{P(z)}-c(z) \equiv 0$, then (5) can be rewritten as

$h(z+\eta) \mathrm{e}^{Q(z+\eta)-Q(z)}-h(z)=\left(h^{\prime}(z)+h(z) Q^{\prime}(z)\right) \mathrm{e}^{P(z)}$.

Next, we discuss two subcases: $1 \leqslant \operatorname{deg} P(z)<$ $\operatorname{deg} Q(z)-1$ and $1 \leqslant \operatorname{deg} P(z)=\operatorname{deg} Q(z)-1$.
Subcase 1.1. Suppose that $1 \leqslant p<q-1$. Then (6) can be rewritten as

$$
\mathrm{e}^{Q(z+\eta)-Q(z)}=\left[1+\left(\frac{h^{\prime}(z)}{h(z)}+Q^{\prime}(z)\right) \mathrm{e}^{P(z)}\right] \frac{h(z)}{h(z+\eta)} .
$$

If $\sigma(h)<1$, since $\operatorname{deg}(Q(z+\eta)-Q(z))=q-1 \geqslant 1$ and $\operatorname{deg} P(z)<q-1$, we know that the order of growth of the left-hand side of (17) is $q-1$, and the order of growth of the right-hand side of (17) is less than $q-1$, a contradiction. Then we have $\sigma(h) \geqslant 1$.

For any given $\varepsilon_{4}, 0<\varepsilon_{3} \leqslant \varepsilon_{4}<\min \left\{\frac{1}{3}(q-\right.$ $\left.\sigma(h)), \frac{1}{3}(q-1-p)\right\}$, there exists $r_{1}>0$ such that for all $z$ satisfying $|z|=r>r_{1}$, we have

$$
\left|\mathrm{e}^{P(z)}\right| \leqslant \exp \left\{r^{p+\varepsilon_{4}}\right\} .
$$

From (17), we see that $\left[1+\left(h^{\prime}(z) / h(z)+\right.\right.$ $\left.\left.Q^{\prime}(z)\right) \mathrm{e}^{P(z)}\right] h(z) / h(z+\eta)$ is an entire function. Then for all $z$ satisfying $|z|=r>r_{1}$ and $|z|=r \notin[0,1] \cup E_{1} \cup E_{2}$, by (6)-(8) and (18), we have

$$
\begin{aligned}
&\left|\left[1+\left(\frac{h^{\prime}(z)}{h(z)}+Q^{\prime}(z)\right) \mathrm{e}^{P(z)}\right] \frac{h(z)}{h(z+\eta)}\right| \\
& \leqslant {\left[1+\left(\left|\frac{h^{\prime}(z)}{h(z)}\right|+\left|Q^{\prime}(z)\right|\right)\left|\mathrm{e}^{P(z)}\right|\right]\left|\frac{h(z)}{h(z+\eta)}\right| } \\
& \leqslant {\left[1+\left(r^{\sigma(h)-1+\varepsilon_{4}}+r^{q-1+\varepsilon_{4}}\right) \exp \left\{r^{p+\varepsilon_{4}}\right\}\right] } \\
& \times \exp \left\{r^{\sigma(h)-1+\varepsilon_{4}}\right\} \\
& \leqslant r^{\sigma(h)+q-2+2 \varepsilon_{4}} \exp \left\{r^{p+\varepsilon_{4}}+r^{\sigma(h)-1+\varepsilon_{4}}\right\} \\
&< \exp \left\{r^{q-1}\right\},
\end{aligned}
$$

that is,

$$
\begin{aligned}
T(r, & {\left.\left[1+\left(\frac{h^{\prime}(z)}{h(z)}+Q^{\prime}(z)\right) \mathrm{e}^{P(z)}\right] \frac{h(z)}{h(z+\eta)}\right) } \\
& =m\left(r,\left[1+\left(\frac{h^{\prime}(z)}{h(z)}+Q^{\prime}(z)\right) \mathrm{e}^{P(z)}\right] \frac{h(z)}{h(z+\eta)}\right) \\
& <r^{q-1} .
\end{aligned}
$$

The above inequality yields

$$
\sigma\left(\left[1+\left(\frac{h^{\prime}(z)}{h(z)}+Q^{\prime}(z)\right) \mathrm{e}^{P(z)}\right] \frac{h(z)}{h(z+\eta)}\right)<q-1 .
$$

It follows from $\operatorname{deg}(Q(z+\eta)-Q(z))=q-1$ that (17) is a contradiction.

Subcase 1.2. Suppose that $1 \leqslant p=q-1$. It follows from (14) that

$$
\left.\begin{array}{rl}
P(z) & =a_{q-1} z^{q-1}+P_{q-2}(z), \\
Q(z+\eta)-Q(z) & =q \eta b_{q} z^{q-1}+Q_{q-2}(z),
\end{array}\right\}
$$


where $a_{q-1}(\neq 0), b_{q}(\neq 0)$ are constants, $P_{q-2}(z)$, $Q_{q-2}(z)$ are polynomials, $\operatorname{deg} P_{q-2}(z) \leqslant q-2$, $\operatorname{deg} Q_{q-2}(z) \leqslant q-2$. In what follows, we consider two subcases: $a_{q-1}=q \eta b_{q}$ and $a_{q-1} \neq q \eta b_{q}$.

Subcase 1.2.1. If $a_{q-1}=q \eta b_{q}$, then (16) can be rewritten as

$$
\mathrm{e}^{-P(z)}=\frac{h(z+\eta)}{h(z)} \mathrm{e}^{Q(z+\eta)-Q(z)-P(z)}-\left[\frac{h^{\prime}(z)}{h(z)}+Q^{\prime}(z)\right] .
$$

It follows from $a_{q-1}=q \eta b_{q}$ that $\operatorname{deg}(Q(z+\eta)-$ $Q(z)-P(z))=\operatorname{deg}\left(Q_{q-2}(z)-P_{q-2}(z)\right) \leqslant q-2$. Using similar reasoning as in the proof of Subcase 1.1, we obtain

$\sigma\left[\frac{h(z+\eta)}{h(z)} \mathrm{e}^{Q(z+\eta)-Q(z)-P(z)}-\left(\frac{h^{\prime}(z)}{h(z)}+Q^{\prime}(z)\right)\right]<q-1$.

It follows from $\operatorname{deg}(-P(z))=q-1 \geqslant 1$ that $(20)$ is a contradiction.

Subcase 1.2.2. If $a_{q-1} \neq q \eta b_{q}$, it follows from (16) and (19) that

$$
\begin{aligned}
& \left(\frac{h^{\prime}(z)}{h(z)}+Q^{\prime}(z)\right) \mathrm{e}^{a_{q-1} z^{q-1}} \\
& =\frac{h(z+\eta)}{h(z)} \mathrm{e}^{q \eta b_{q} z^{q^{-1}}+Q_{q-2}(z)-P_{q-2}(z)}-\mathrm{e}^{-P_{q-2}(z)} .
\end{aligned}
$$

Without loss of generality, we assume that $q\left|\eta b_{q}\right| \leqslant$ $\left|a_{q-1}\right|$. Set $\arg a_{q-1}=\theta_{1}$ and $\arg \left(\eta b_{q}\right)=\theta_{2}$. For the above given $\varepsilon_{3}$ and for all $z$ satisfying $|z|=r>r_{2}$ and $|z|=r \notin[0,1] \cup E_{1} \cup E_{2}, z=r \mathrm{e}^{\mathrm{i} \theta_{0}}$, where $\theta_{0}$ is a real constant such that $\cos \left((q-1) \theta_{0}+\theta_{1}\right)=1$, by (6)-(8), we have

$$
\begin{aligned}
\left|\left[\frac{h^{\prime}(z)}{h(z)}+Q^{\prime}(z)\right] \mathrm{e}^{a_{q-1} z^{q-1}}\right| \\
\quad \geqslant\left[\left|Q^{\prime}(z)\right|-\left|\frac{h^{\prime}(z)}{h(z)}\right|\right]\left|\mathrm{e}^{a_{q-1} z^{q-1}}\right| \\
\quad \geqslant\left(r^{q-1-\varepsilon_{3}}-r^{\sigma(h)-1+\varepsilon_{3}}\right) \exp \left\{\left|a_{q-1}\right| r^{q-1}\right\} \\
\quad \geqslant r^{q-1-2 \varepsilon_{3}}(1+o(1)) \exp \left\{\left|a_{q-1}\right| r^{q-1}\right\} \\
\quad \geqslant \exp \left\{\left|a_{q-1}\right| r^{q-1}\right\},
\end{aligned}
$$

and

$$
\begin{aligned}
& \left|\frac{h(z+\eta)}{h(z)} \mathrm{e}^{q \eta b_{q} z^{q-1}+Q_{q-2}(z)-P_{q-2}(z)}-\mathrm{e}^{-P_{q-2}(z)}\right| \\
& \leqslant\left|\frac{h(z+\eta)}{h(z)}\right|\left|\mathrm{e}^{q \eta b_{q} z^{q-1}+Q_{q-2}(z)-P_{q-2}(z)}\right|+\left|\mathrm{e}^{-P_{q-2}(z)}\right| \\
& \leqslant \exp \left\{r^{\sigma(h)-1+\varepsilon_{3}}\right\} \\
& \quad \times \exp \left\{q\left|\eta b_{q}\right| \cos \left((q-1) \theta_{0}+\theta_{2}\right) r^{q-1}+O\left(r^{q-2}\right)\right\} \\
& \leqslant \exp \left\{q\left|\eta b_{q}\right| \cos \left((q-1) \theta_{0}+\theta_{2}\right) r^{q-1}+o\left(r^{q-1}\right)\right\},
\end{aligned}
$$

that is,

$$
\begin{aligned}
& \exp \left\{\left|a_{q-1}\right| r^{q-1}\right\} \\
& \quad \leqslant \exp \left\{q\left|\eta b_{q}\right| \cos \left((q-1) \theta_{0}+\theta_{2}\right) r^{q-1}+o\left(r^{q-1}\right)\right\} .
\end{aligned}
$$

We claim that $q\left|\eta b_{q}\right| \cos \left((q-1) \theta_{0}+\theta_{2}\right)<\left|a_{q-1}\right|$. In fact, if $q\left|\eta b_{q}\right|=\left|a_{q-1}\right|$, it follows from $a_{q-1} \neq q \eta b_{q}$ that $\cos \left((q-1) \theta_{0}+\theta_{2}\right) \neq 1$, then $\cos \left((q-1) \theta_{0}+\theta_{2}\right)<$ 1. Thus $q\left|\eta b_{q}\right| \cos \left((q-1) \theta_{0}+\theta_{2}\right)<q\left|\eta b_{q}\right|=\left|a_{q-1}\right|$. If $q\left|\eta b_{q}\right|<\left|a_{q-1}\right|$, then $q\left|\eta b_{q}\right| \cos \left((q-1) \theta_{0}+\theta_{2}\right) \leqslant$ $q\left|\eta b_{q}\right|<\left|a_{q-1}\right|$. For any given $\varepsilon_{5}, 0<\varepsilon_{5}<\frac{1}{3}\left(\left|a_{q-1}\right|-\right.$ $\left.q\left|\eta b_{q}\right| \cos \left((q-1) \theta_{0}+\theta_{2}\right)\right)$, it follows from (22) that

$$
\begin{aligned}
& \exp \left\{\left|a_{q-1}\right| r^{q-1}\right\} \\
& \quad \leqslant \exp \left\{q\left|\eta b_{q}\right| \cos \left((q-1) \theta_{0}+\theta_{2}\right) r^{q-1}+o\left(r^{q-1}\right)\right\} \\
& \quad<\exp \left\{\left(\left|a_{q-1}\right|-\varepsilon_{5}\right) r^{q-1}\right\} .
\end{aligned}
$$

This is a contradiction.

Case 2. Suppose that $p=q$. For $a_{q}$ and $b_{q}$, we consider three subcases: (2.1) $a_{q}=b_{q}$; (2.2) $a_{q}=$ $-b_{q} ;(2.3) a_{q} \neq b_{q}$ and $a_{q} \neq-b_{q}$.

Subcase 2.1. Suppose that $a_{q}=b_{q}$. Then (13) can be rewritten as

$$
\begin{aligned}
& \left(h^{\prime}(z)+h(z) Q^{\prime}(z)\right) \mathrm{e}^{P(z)}-c(z) \mathrm{e}^{-Q(z)} \\
& \quad=h(z+\eta) \mathrm{e}^{Q(z+\eta)-Q(z)}-h(z)-d(z) \mathrm{e}^{P(z)-Q(z) .} .
\end{aligned}
$$

Since $\sigma(h)<q, \operatorname{deg}(Q(z+\eta)-Q(z))=q-1$, $\max \{\sigma(c), \sigma(d)\}<q$ and $\operatorname{deg}(P(z)-Q(z)) \leqslant q-$ 1 , we have $\sigma\left(h^{\prime}(z)+h(z) Q^{\prime}(z)\right)<q$ and $\sigma(h(z+$

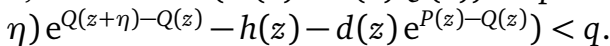

Noting that $\mathrm{e}^{P(z)}, \mathrm{e}^{-Q(z)}$ and $\mathrm{e}^{P(z)+Q(z)}$ are of regular growth, and $\sigma\left(\mathrm{e}^{P(z)}\right)=\sigma\left(\mathrm{e}^{-Q(z)}\right)=$ $\sigma\left(\mathrm{e}^{P(z)+Q(z)}\right)=q$, it follows from Lemma 4 and (23) that

$$
h^{\prime}(z)+h(z) Q^{\prime}(z) \equiv 0 .
$$

If $h^{\prime}(z)+h(z) Q^{\prime}(z) \equiv 0$, suppose that $h(z)$ is a polynomial. Then $h(z) \equiv 0$, it contradicts $h(z) \not \equiv$ 0 . If $h^{\prime}(z)+h(z) Q^{\prime}(z) \equiv 0$, suppose that $h(z)$ is a transcendental entire function. Then $h(z)=c \mathrm{e}^{-Q(z)}$, $c \in \mathbb{C} \backslash\{0\}$, that is, $\sigma(h)=q$, it contradicts $\sigma(h)<q$. Then we see that $h^{\prime}(z)+h(z) Q^{\prime}(z) \equiv 0$ is absurd.

Subcase 2.2. Suppose that $a_{q}=-b_{q}$. Then (13) can be rewritten as

$$
\begin{aligned}
& {\left[\left(h^{\prime}(z)+h(z) Q^{\prime}(z)\right) \mathrm{e}^{P(z)+Q(z)}-c(z)\right] \mathrm{e}^{-Q(z)}} \\
& \quad+d(z) \mathrm{e}^{P(z)-Q(z)}=h(z+\eta) \mathrm{e}^{Q(z+\eta)-Q(z)}-h(z) .
\end{aligned}
$$


Since $\sigma(h)<q, \operatorname{deg}(Q(z+\eta)-Q(z))=q-1$, $\max \{\sigma(c), \sigma(d)\}<q$ and $\operatorname{deg}(P(z)+Q(z)) \leqslant q-1$, we have $\sigma\left(\left(h^{\prime}(z)+h(z) Q^{\prime}(z)\right) \mathrm{e}^{P(z)+Q(z)}-c(z)\right)<q$ and $\sigma\left(h(z+\eta) \mathrm{e}^{Q(z+\eta)-Q(z)}-h(z)\right)<q$.

Noting that $\mathrm{e}^{-Q(z)}, \mathrm{e}^{P(z)-Q(z)}$ and $\mathrm{e}^{-P(z)}$ are of regular growth, and $\sigma\left(\mathrm{e}^{-Q(z)}\right)=\sigma\left(\mathrm{e}^{-P(z)}\right)=$ $\sigma\left(\mathrm{e}^{P(z)-Q(z)}\right)=q$, it follows from Lemma 4 and (24) that

$$
h(z+\eta) \mathrm{e}^{Q(z+\eta)-Q(z)}-h(z) \equiv 0 .
$$

Making use of the above identity, we obtain

$$
\mathrm{e}^{Q(z)-Q(z+\eta)} \equiv \frac{h(z+\eta)}{h(z)} .
$$

Combining with (7) and (2), we conclude that the order of growth of the left-hand side of (25) is $q-1$, and the order of growth of the right-hand side of (25) is less than $q-1$, a contradiction.

Subcase 2.3. Suppose that $a_{q} \neq b_{q}$ and $a_{q} \neq$ $-b_{q}$. Then (13) can be rewritten as

$$
\begin{array}{r}
\left(h^{\prime}(z)+h(z) Q^{\prime}(z)\right) \mathrm{e}^{P(z)}-c(z) \mathrm{e}^{-Q(z)}+d(z) \mathrm{e}^{P(z)-Q(z)} \\
=h(z+\eta) \mathrm{e}^{Q(z+\eta)-Q(z)}-h(z) . \quad
\end{array}
$$

Since $\sigma(h)<q, \operatorname{deg}(Q(z+\eta)-Q(z))=q-1$ and $\max \{\sigma(c), \sigma(d)\}<q$, we have $\sigma\left(h^{\prime}(z)+\right.$ $\left.h(z) Q^{\prime}(z)\right)<q$ and $\sigma\left(h(z+\eta) \mathrm{e}^{Q(z+\eta)-Q(z)}-h(z)\right)<q$.

Noting that $\mathrm{e}^{ \pm P(z)}, \mathrm{e}^{ \pm Q(z)}$ and $\mathrm{e}^{P(z) \pm Q(z)}$ are of regular growth, and $\sigma\left(\mathrm{e}^{ \pm P(z)}\right)=\sigma\left(\mathrm{e}^{ \pm Q(z)}\right)=$ $\sigma\left(\mathrm{e}^{P(z) \pm Q(z)}\right)=q$, it follows from Lemma 4 and (26) that

$$
\begin{aligned}
h^{\prime}(z)+h(z) Q^{\prime}(z) & \equiv 0, \\
h(z+\eta) \mathrm{e}^{Q(z+\eta)-Q(z)}-h(z) & \equiv 0 .
\end{aligned}
$$

Using similar reasoning as above, we also obtain a contradiction.

Thus $P(z)$ can only be a constant, so is $\mathrm{e}^{P(z)}$. Set $\mathrm{e}^{P(z)} \equiv A$, where $A$ is a non-zero constant. It follows from (11) that

$$
\frac{\Delta_{\eta} f(z)-a(z)}{f^{\prime}(z)-a(z)}=A .
$$

By Lemma 5, we have

$$
f(z)=b(z)+H(z) \mathrm{e}^{c z},
$$

where $b(z)$ is an entire function with $\sigma(b)<1$, $H(z) \not \equiv 0$ is an entire function with $\lambda(H)=\sigma(H)<1$ and $A, c, \eta \in \mathbb{C} \backslash\{0\}$ are constants satisfying $\mathrm{e}^{c \eta}=$ $1+A c$. This completes the proof of Theorem 6 .
Acknowledgements: This study was partly supported by the National Natural Science Foundation for Young Scientists of China (Nos: 11701524, 11801093) and by the National Natural Science Foundation of Guangdong Province (No: 2016A030313686). The authors would like to thank the referee for their thorough reviewing with constructive suggestions and comments to the paper.

\section{REFERENCES}

1. Hayman WK (1964) Meromorphic Function, Clarendon Press, Oxford.

2. Laine I (1993) Nevanlinna Theory and Complex Differential Equations, De Gruyter, Berlin.

3. Yang L (1993) Value Distribution Theory, SpringerVerlag, Berlin-Heidelberg.

4. Yang CC, Yi HX (2003) Uniqueness Theory of Meromorphic Functions, Kluwer Academic, Dordrecht.

5. Brück R (1996) On entire functions which share one value CM with their first derivative. Results Math $\mathbf{3 0}$, $21-24$.

6. Gundersen G, Yang LZ (1998) Entire functions that share one value with one or two of their derivatives. J Math Anal Appl 223, 88-95.

7. Chen ZX, Yi HY (2013) On sharing values of meromorphic functions and their differences. Results Math 63, 557-565.

8. Chen ZX (2014) On the difference counterpart of Brück's conjecture. Acta Math Sci 34, 653-659.

9. Heittokangas J, Korhonen R, Laine I, Rieppo J, Zhang $J$ (2009) Value sharing results for shifts of meromorphic functions and sufficient conditions for perodicity. J Math Anal Appl 355, 352-363.

10. Li S, Gao ZS (2011) Entire functions sharing one or two finite values CM with their shifts or difference operator. Arch Math 97, 475-483.

11. Li S, Gao ZS (2012) Erratum to: A note on Brück's conjecture. Arch Math 99, 255-259.

12. Li XM, Yi HX (2014) Entire functions sharing an entire function of smaller order with their difference operator. Acta Math Sin English Series 30, 481-498.

13. Liu K, Dong XJ (2014) Some results related to complex differential-difference equations of certain types. Bull Korean Math Soc 51, 1453-1467.

14. Chen MF, Gao ZS (2017) Some results on complex differential-difference analogue of Brück conjecture. Commun Korean Math Soc 32, 361-373.

15. Gundersen G (1988) Estimates for the logarithmic derivative of a meromorphic function, plus similar estimates. J London Math Soc 37, 88-104.

16. Chiang YM, Feng SJ (2008) On the Nevanlinna characteristic of $f(z+\eta)$ and difference equations in the complex plane. Ramanujan J 16, 105-129.

17. Hayman WK (1960) Slowly growing integral and subharmonic functions. Comment Math Helv 34, 75-84.

18. Bergweiler W, Langlely JK (2007) Zeros of difference of meromorphic functions. Math Proc Cambridge Philos Soc 142, 133-147. 